\title{
Seeking a reaction
}

Immunological reagents and techniques.

Monoclonal Production

Invitrogen

www.invitrogen.com

GIBCO products showcased

This free brochure from Invitrogen details the company's latest offerings for cell culture. It includes descriptions of GIBCO products for monoclonal antibody production: Chinese hamster ovary $(\mathrm{CHO})$ cells, used for the expression of monoclonal antibodies or recombinant proteins intended for human therapeutic or diagnostic applications; $\mathrm{CHO}$ media, serum-free formulations for use in large-scale suspension culture applications; cholesterol lipid concentrate and CD Hybridoma Medium, a system used to grow NSO cells to high density; hybridoma-SFM, a serum-free medium used to support a range of hybridoma cell lines in a variety of culture systems; and OptiMAb Monoclonal Antibody Production Enhancer, a protein-free concentrate used to increase Mab production. The brochure is also available online at www.invitrogen.com.

\section{$\mu$ MACS with Protein MicroBeads}

Miltenyi Biotec www.miltenyibiotec.com Immunoprecipitation in a Flash

Miltenyi Biotec's website now contains an animated demonstration of immunoprecipitation using the company's $\mu$ MACS and Protein A or G Microbeads. It demonstrates, using the latest in graphics, the principle by which immunoprecipitation can be achieved in under two hours with the $\mu$ MACS system.

\section{Autokit C3}

Wako www.wakousa.com

Fishing for complements

The Autokit C3 is an assay kit for the quantitative determination of complement $\mathrm{C} 3$ in human serum or plasma. The complement system is a plasma-based protein cascade that functions as a highly regulated and effective immune barrier. It is comprised of more than 30 membrane bound and plasma

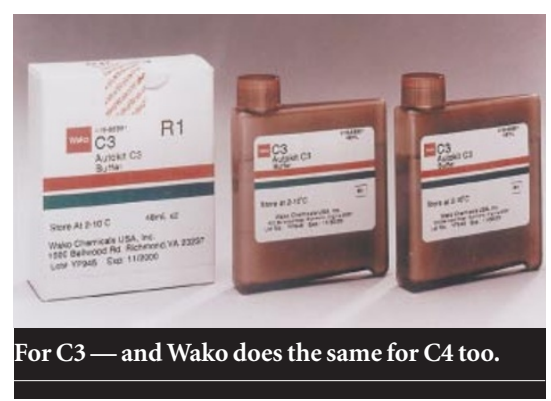

components that work in concert to promote a variety of immune functions. $\mathrm{C} 3$ is central to all complement pathways and the most abundant circulating complement protein. Measurement of C3 is an important index for the diagnosis of immune disease such as lupus nephritis, allergies and inflammation. It is also used to monitor fulminant liver diseases.

\section{Immunology catalogue}

Bender MedSystems

www.bendermedsystems.com

Novel reading

Bender MedSystems' latest catalogue features a number of new technologies, including fluorescent bead immunoassays for multiple cytokine detection and instant ELISAs, as well as recombinant proteins and reagents for growth factors. Products used in cell adhesion, apoptosis, cytokines/growth factors and tumour biology work with several different species, including human, mouse, rat, monkey and pig, are included. There is also a reference section with technical information on methods.

\section{STAT-IV}

Taconic Biotechnology www.taconic.com Ascite alternative

STAT- $I V$ is a process technology for in vitro production of monoclonal antibodies. The Integra Biosciences CELLine CK 1000 device can produce up to $200 \mathrm{mg}$ of antibody per 42-day run. Target amounts of antibody ranging from from $100 \mathrm{mg}$ to $2 \mathrm{~g}$ can be obtained. Production from hybridomas of any origin (mouse, rat or hamster) is possible. A high density of cells with a low media volume results in high concentrations of antibody in solution. The device complies with IACUC recommendations for those seeking alternatives to ascites production.

\section{Automated antigen retrieval}

BioGenex www.biogenex.com Standardized results at the touch of a button

Antigen retrieval can produce improved staining for a wide range of monoclonal and polyclonal antibodies. Its use helps to overcome false negative staining of overfixed tissue, expands the range of antibodies useful for routinely processed tissue and increases the usefulness of archival materials for retrospective studies. The i1000 automated antigen retrieval system eliminates the variability often encountered in antigen retrieval and produces standardized results.

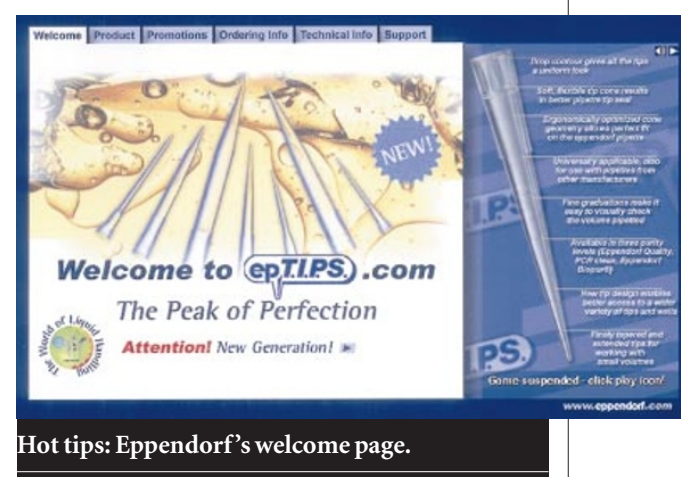

epTIPS online

Eppendorf www.eptips.com

Means to an end

Eppendorf has launched a new homepage containing information on its newest range of pipette tips. The website, eptips.com, provides technical information, ordering numbers and packaging units, as well as customer support and promotions. Customers in Western Europe can order samples online at no cost.

These notes are compiled in the Nature office from information provided by the manufacturers. Press releases to be considered for publication can be sent to newproducts@nature.com. 